\title{
Correction to: Safety and efficacy of preoperative chemotherapy followed by esophagectomy versus upfront surgery for resectable esophageal squamous cell carcinoma
}

\author{
Takanori Kurogochi ${ }^{1} \cdot$ Michitaka Honda $^{1,2} \cdot$ Kotaro Yamashita $^{1} \cdot$ Masaru Hayami ${ }^{1}$. Akihiko Okamura ${ }^{1}$ Yu Imamura ${ }^{1}$. \\ Shinji Mine ${ }^{1} \cdot$ Masayuki Watanabe $^{1}$
}

Published online: 31 March 2020

(c) Springer Nature Singapore Pte Ltd. 2020

\section{Correction to: Surgery Today (2019) 49:150-157 https://doi.org/10.1007/s00595-018-1718-8}

In the original publication, in Abstract, the second sentence of Results has been incorrectly published as:

Complications occurred in $76(68.5 \%)$ and $76(64.4 \%)$ patients in NAC and US groups, respectively $(p=0.51)$, and severe complications occurred in 17 (22.4\%) and 30 (39.5\%) patients, respectively $(p=0.057)$.

The correct sentence is given below:

Complications occurred in 76 (68.5\%) and 76 (64.4\%) patients in NAC and US groups, respectively $(p=0.51)$, and severe complications occurred in 17 (22.4\%) and 30 (25.4\%) patients, respectively $(p=0.057)$.

Under the section: "Surgical procedures and outcomes", the sixth sentence has been incorrectly published as:

The incidences of severe complications of Clavien-Dindo classification grade IIIa or higher were $22.4 \%(n=17)$ in the NAC group and $39.5 \%(n=30)$ in the US group $(p=0.057)$.

The correct sentence is given below:

The incidences of severe complications of Clavien-Dindo classification grade IIIa or higher were $15.3 \%(n=17)$ in the NAC group and $25.4 \%(n=30)$ in the US group $(p=0.057)$.

The original article can be found online at https://doi.org/10.1007/ s00595-018-1718-8.

Masayuki Watanabe

sayuki.watanabe@jfcr.or.jp

1 Department of Gastroenterological Surgery, The Cancer Institute Hospital of Japanese Foundation for Cancer Research, 3-8-31 Ariak, Koto-ku, Tokyo 135-8550, Japan

2 Department of Minimally Invasive Surgical and Medical Oncology, Fukushima Medical University, Fukushima, Japan 